\title{
Orbit- and atom-resolved spin textures of intrinsic, extrinsic, and hybridized Dirac cone states
}

\author{
Lin Miao, ${ }^{1, *}$ Z. F. Wang, ${ }^{2, *}$ Meng-Yu Yao, ${ }^{1}$ Fengfeng Zhu, ${ }^{1}$ J. H. Dil,${ }^{3,4}$ C. L. Gao, ${ }^{1}$ Canhua Liu, ${ }^{1}$ Feng Liu,,${ }^{2,}$ \\ Dong Qian,,$^{1, \dagger}$ and Jin-Feng Jia ${ }^{1, \dagger}$ \\ ${ }^{1}$ Key Laboratory of Artificial Structures and Quantum Control (Ministry of Education), Department of Physics and Astronomy, \\ Shanghai Jiao Tong University, Shanghai 200240, China \\ ${ }^{2}$ Department of Materials Science and Engineering, University of Utah, Salt Lake City, Utah 84112, USA \\ ${ }^{3}$ Swiss Light Source, Paul Scherrer Institute, CH-5232 Villigen, Switzerland \\ ${ }^{4}$ Physik-Institut, Universität Zürich-Irchel, 8057 Zürich, Switzerland
}

(Received 13 September 2013; revised manuscript received 15 March 2014; published 14 April 2014)

\begin{abstract}
Combining first-principles calculations and spin- and angle-resolved photoemission spectroscopy measurements, we identify the helical spin textures for three different Dirac cone states in the interfaced systems of a two-dimensional (2D) topological insulator (TI) of a $\mathrm{Bi}(111)$ bilayer and a three-dimensional (3D) $\mathrm{TI} \mathrm{Bi}_{2} \mathrm{Se}_{3}$ or $\mathrm{Bi}_{2} \mathrm{Te}_{3}$. The spin texture is found to be the same for the intrinsic Dirac cone of $\mathrm{Bi}_{2} \mathrm{Se}_{3}$ or $\mathrm{Bi}_{2} \mathrm{Te}_{3}$ surface states, the extrinsic Dirac cone of Bi bilayer induced by the Rashba effect, and the hybridized Dirac cone between the former two states. Further orbit- and atom-resolved analysis shows that $s$ and $p_{z}$ orbits have a clockwise (counterclockwise) spin rotation tangent to the iso-energy contour of the upper (lower) Dirac cone, while $p_{x}$ and $p_{y}$ orbits have radial spin components. The Dirac cone states may reside on different atomic layers, but have the same spin texture. Our results suggest that the unique spin texture of Dirac cone states is a signature property of spin-orbit coupling, independent of topology.
\end{abstract}

DOI: 10.1103/PhysRevB.89.155116

PACS number(s): 73.20.-r, 73.22.-f, 75.70.Tj

One outstanding property that 3D TIs possess is the helical Dirac cone surface state residing inside a bulk gap, in which electron spin is locked with momentum [1,2]. This spinmomentum locking property makes TIs promising materials for realizing spintronic devices, because the electron backscattering is forbidden for nonmagnetic impurities. Therefore, understanding the spin texture of helical Dirac cone states is of fundamental interest with practical implication.

Experimentally, the helical spin texture of surface Dirac cone state of 3D TIs has been directly detected by spinand angle-resolved photoemission spectroscopy (SARPES) [3-8]. It can also be manipulated through its interaction with polarized light $[9,10]$. Very recently it has been noted that because of the strong spin-orbit coupling (SOC), total angular momentum, spin plus orbital angular momentum, should be the good quantum number in TIs. Consequently, the spin texture of TIs is expected to be coupled with atomic orbits in a specific manner [11-13]. Both theory [11] and experiment [12,13] have shown some very interesting patterns of radial/tangential spin texture coupled with $p$ orbits in the $\mathrm{Bi}_{2} \mathrm{Se}_{3}$ family.

Besides the "intrinsic" Dirac cone state of TIs, the "extrinsic" Dirac cone state may be generated by Rashba effect, such as the one formed when the $\mathrm{Bi}(111)$ bilayer is grown on the $\mathrm{Bi}_{2} \mathrm{Se}_{3}$ substrate [14,15]. Also, when the $\mathrm{Bi}(111)$ bilayer is grown on $\mathrm{Bi}_{2} \mathrm{Te}_{3}[14,16]$, a kind of hybridized Dirac cone state is formed between the intrinsic surface Dirac cone state of $\mathrm{Bi}_{2} \mathrm{Te}_{3}$ and the extrinsic Rashba Dirac cone state of $\mathrm{Bi}(111)$. These Dirac cone states are all associated with strong SOC. However, different from the intrinsic surface states of TI, which are topologically protected inside a bulk gap, the extrinsic and hybridized states have no topological origin, even though they

\footnotetext{
*L.M. and Z.F.W. contributed equally to this work.

†Corresponding authors: fliu@eng.utah.edu; dqian@sjtu.edu.cn; jfjia@sjtu.edu.cn
}

are formed by interfacing a 2D TI of $\mathrm{Bi}(111)$ bilayer [17-21] and a $3 \mathrm{D}$ TI of $\mathrm{Bi}_{2} \mathrm{Se}_{3}$ or $\mathrm{Bi}_{2} \mathrm{Te}_{3}$. It will be interesting to find out the spin texture of these nontopological Dirac states, in comparison with the topological surface Dirac states of 3D TIs.

In this work, the spin texture of three different Dirac cone states in the system of $\mathrm{Bi} / \mathrm{Bi}_{2} \mathrm{Se}_{3}$ and $\mathrm{Bi} / \mathrm{Bi}_{2} \mathrm{Te}_{3}$ are systemically studied. Both first-principles calculations and SARPES measurements show that the total spins form an identical helical spin texture for all three Dirac cones. Furthermore, the orbit-resolved calculations reveal that $s$ and $p_{z}$ orbits have a clockwise (counterclockwise) spin rotation tangent to the iso-energy contour of the upper (lower) Dirac cone, while $p_{x}$ and $p_{y}$ orbits have a radial spin component, in agreement with recent studies [11-13]. The atom-resolved calculations reveal that the Dirac cone states may reside on different atomic layers, but their spin texture remains the same.

Our first-principles calculations are carried out in the framework of generalized gradient approximation with a PerdewBurke-Ernzerhof functional using the VASP package [22]. The lattice constants of the substrate are taken from experiments ( $a=4.138 \AA$ for $\mathrm{Bi}_{2} \mathrm{Se}_{3}$ and $a=4.386 \AA$ for $\left.\mathrm{Bi}_{2} \mathrm{Te}_{3}\right)$, and the $\mathrm{Bi}(111)$ bilayer is strained to match the substrate lattice parameter. All calculations are performed with a plane-wave cutoff of $400 \mathrm{eV}$ on an $11 \times 11 \times 1$ Monkhorst-pack $k$-point mesh. The substrate is modeled by a slab of 6 quintuple layer (QL) $\mathrm{Bi}_{2} \mathrm{Se}_{3}$ and $\mathrm{Bi}_{2} \mathrm{Te}_{3}$ because it takes 5-6 QLs to eliminate top-bottom surface interaction for the film to assume bulk property [23], and the vacuum layers are over $20 \AA$ thick to ensure decoupling between neighboring slabs. We have also done test calculations using $8 \mathrm{QLs}$ of $\mathrm{Bi}_{2} \mathrm{Se}_{3}$, which confirm that our results using $6 \mathrm{QLs}$ are converged. During structural relaxation, atoms in the lower $4 \mathrm{QL}$ substrate are fixed in their respective bulk positions, and the Bi bilayer and upper $2 \mathrm{QL}$ of substrate are allowed to relax until the forces are smaller than $0.01 \mathrm{eV} / \AA$. 

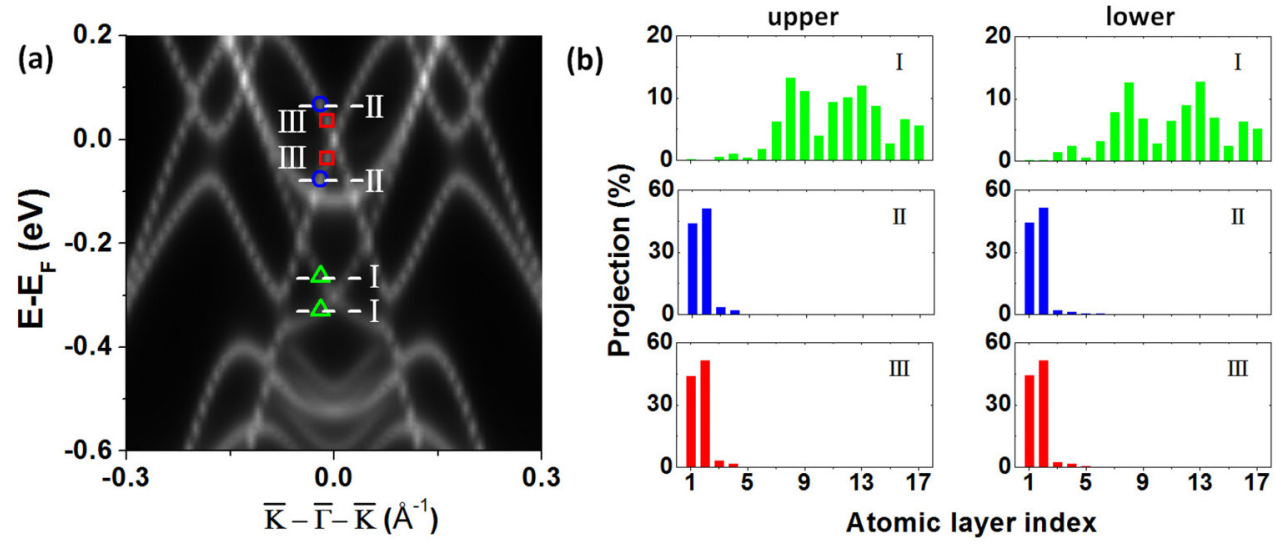

(c)

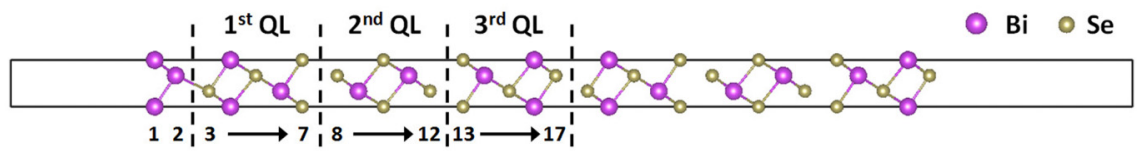

(d)
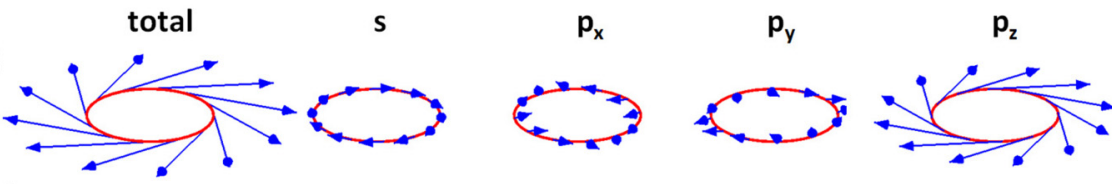

$p_{x}+p_{y}$

I
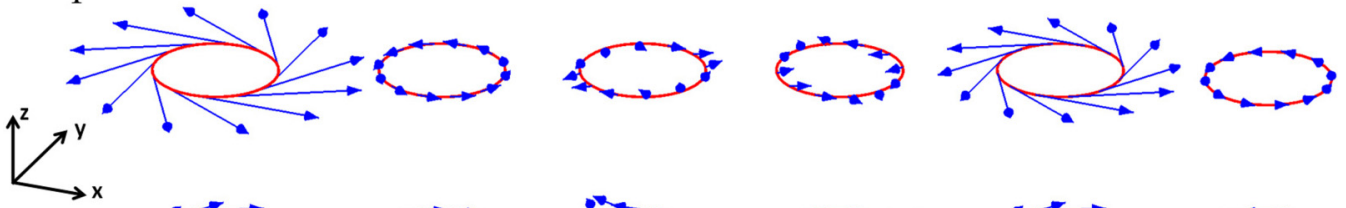

(e)
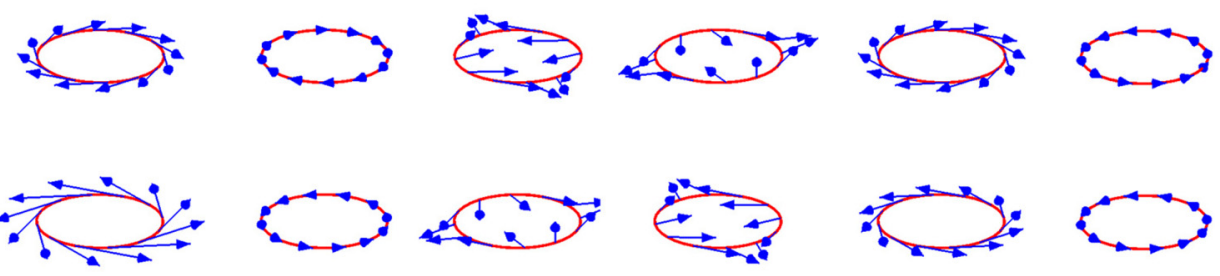

II
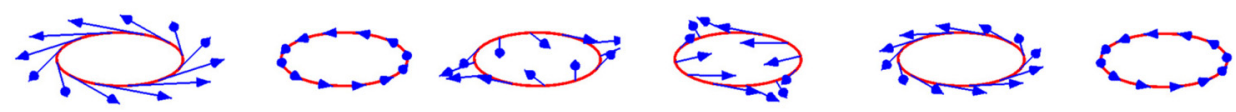

FIG. 1. (Color online) (a) $\mathrm{Bi} / \mathrm{Bi}_{2} \mathrm{Se}_{3}$ spectral function projected onto a top Bi bilayer plus upper $2 \mathrm{QL} \mathrm{Bi}_{2} \mathrm{Se}_{3}$. (b) Percentage contribution of different atoms to the Dirac cone states marked by labels I, II, and III with different colors and symbols in (a). Upper (lower) denotes upper (lower) Dirac cone states and the atomic layer index is shown in (c). (c) Atomic structure of the optimized $\mathrm{Bi} / \mathrm{Bi}_{2} \mathrm{Se}_{3}$. $\mathrm{A} \mathrm{Bi}$ bilayer and upper $3 \mathrm{QL} \mathrm{Bi}_{2} \mathrm{Se}_{3}$ are labeled with numbers to denote their atomic layer index. (d) and (e) Orbit-resolved spin textures of the iso-energy contour Dirac cone state at the energy marked by labels I and II in (a).

First, we take a look at the $\mathrm{Bi} / \mathrm{Bi}_{2} \mathrm{Se}_{3}$, where both intrinsic and extrinsic Dirac cone states coexist [14]. Figure 1(a) shows the spectral function of $\mathrm{Bi} / \mathrm{Bi}_{2} \mathrm{Se}_{3}$ projected onto the Bi bilayer plus top $2 \mathrm{QL}$ of $\mathrm{Bi}_{2} \mathrm{Se}_{3}$. One sees two Dirac cones at the $\Gamma$ point, one below and the other near the Fermi level. To reveal the origin of these two Dirac cones, we plot their real-space distribution by choosing three data points from the upper and lower Dirac cones, as marked by I, II, and III in Fig. 1(a). The corresponding momenta are $-0.02,-0.02$, and $-0.01 \AA^{-1}$ for I, II, and III states, respectively. The atom-projected state components are shown in Fig. 1(b), with the atomic layer indexes labeled in Fig. 1(c). The Dirac cone states below the Fermi level come almost completely from the substrate with little contribution from the Bi bilayer [Fig. 1(b), top panel]. This indicates that it is an intrinsic Dirac cone of $\mathrm{Bi}_{2} \mathrm{Se}_{3}$. The Dirac cone states near the Fermi level come predominantly from the top Bi bilayer, having little substrate contribution [see Fig. 1(b), two bottom panels]. This indicates that it is an extrinsic Dirac cone generated by the Rashba splitting of $\mathrm{Bi}$ bilayer bands, as discussed before [14,15]. Additionally, we note that due to interfacial interaction with the Bi bilayer, the intrinsic surface Dirac cone has a wide real-space distribution in the second and third QL [Fig. 1(b), top panel] that peaks at the boundary between the first and second, and the second and third QL [i.e., the seventh and eighth, and 12th and 13th atomic layer as seen in Fig. 1(c)]. This is different from the surface Dirac cone of bare $\mathrm{Bi}_{2} \mathrm{Se}_{3}$ that decays from the first and second QL [24]. The Bi bilayer pushes down and broadens the surface states of $\mathrm{Bi}_{2} \mathrm{Se}_{3}$. This is consistent with the recent findings by $\mathrm{Wu}$ et al. that the vertical location of the surface Dirac cone state of a 3D TI can be tuned by depositing a layer of conventional insulator [25].

Although the two Dirac cones in Fig. 1(a) have different physical origins, their spin textures are found to be the same. 

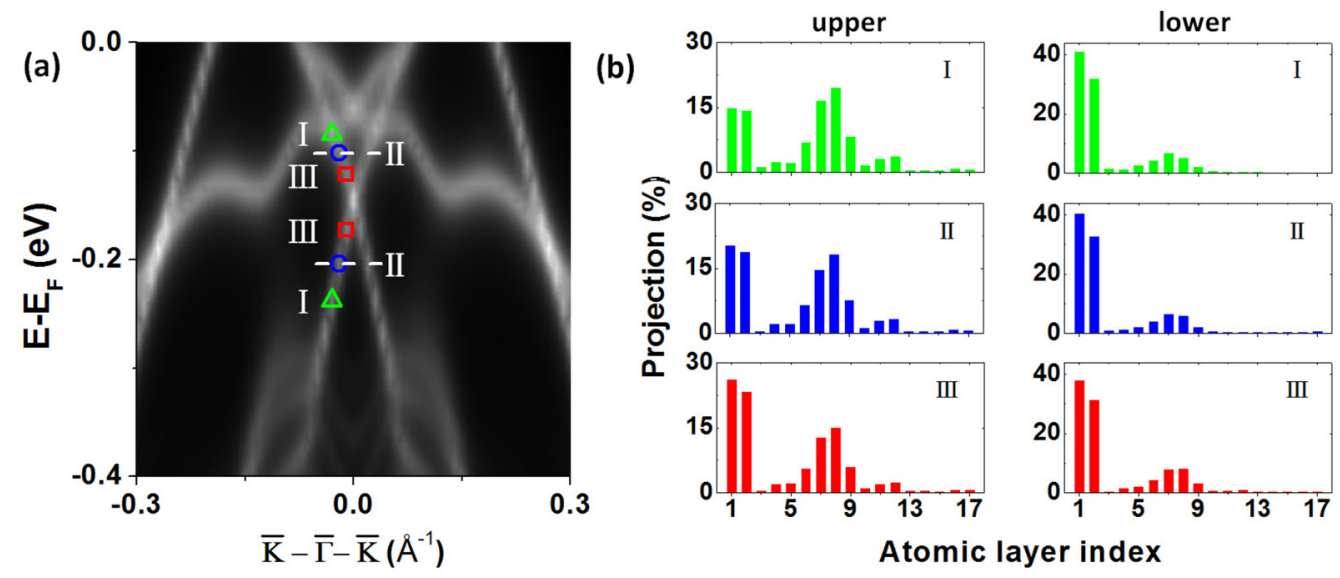

(c)
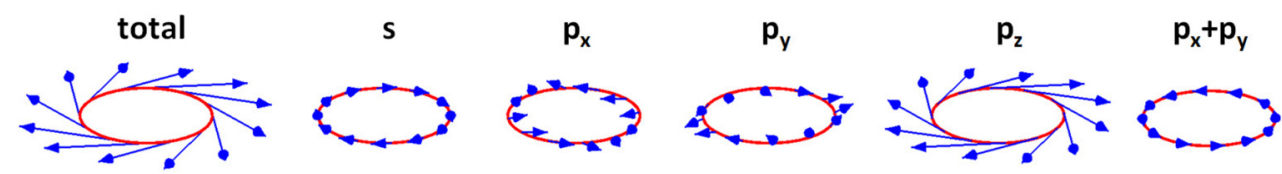

II
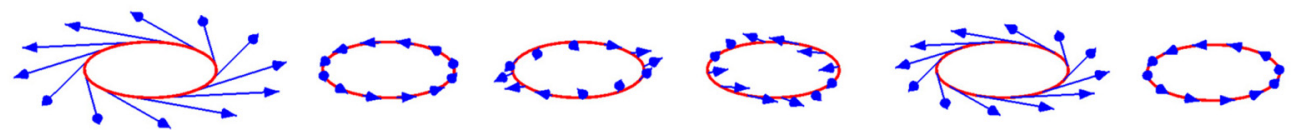

FIG. 2. (Color online) (a) $\mathrm{Bi} / \mathrm{Bi}_{2} \mathrm{Te}_{3}$ spectral function projected onto the top Bi bilayer plus upper $1 \mathrm{QL} \mathrm{Bi}_{2} \mathrm{Te}_{3}$. (b) Percentage contribution of different atoms to the Dirac cone states marked by labels I, II, and III with different colors and symbols in (a). (c) Orbit-resolved spin textures of the iso-energy contour Dirac cone state at the energy marked by label II in (a). The scaling factor of the arrow length is the same as that in Fig. 1.

The total and orbit-resolved spin textures along the iso-energy contour at the energy marked by $\mathrm{I}$ (intrinsic $\mathrm{Bi}_{2} \mathrm{Se}_{3}$ Dirac cone) and II (extrinsic Bi Dirac cone) are shown in Figs. 1(d) and 1(e), respectively. The total spins of both Dirac cones are oriented clockwise (counterclockwise) in the upper (lower) Dirac cone. $s$ and $p_{z}$ orbits have the same helical spin texture as the total spin, but $p_{x}$ and $p_{y}$ orbits have a different spin texture with additional radial spin component, pointing toward opposite directions along the $135^{\circ}$ or $45^{\circ}$ axis. In addition, $p_{x}$ orbit spin texture in the upper (lower) Dirac cone is the same as $p_{y}$ orbit spin texture in the lower (upper) Dirac cone. These in-plane $p$ orbit spin textures are consistent with the theoretical model prediction by Zhang et al. [11]. We note that although both $p_{x}$ and $p_{y}$ orbit spins have radial components, their summation, $p_{x}+p_{y}$ orbit spins is helical becoming tangent to iso-energy contour, albeit they are oriented counterclockwise in both upper and lower Dirac cones [see Figs. 1(d) and 1(e)]. The total helical in-plane $p$ orbit spins with the same orientation in the upper and lower Dirac cones have been recently experimentally observed in the $\mathrm{Bi}_{2} \mathrm{Se}_{3}$ surface Dirac cones [13]. The only difference between the spin textures of intrinsic and extrinsic Dirac cones is the spin magnitude. For the intrinsic Dirac cone, the amplitude of $s, p_{x}$, and $p_{y}$ orbit spins are negligible, and the magnitude of $p_{z}$ orbit spin is the largest. While for the extrinsic Dirac cone, the amplitude of $s$ orbit spin is negligible, and the magnitude of three $p$ orbit spins are comparable to each other.

Next, we turn to the $\mathrm{Bi} / \mathrm{Bi}_{2} \mathrm{Te}_{3}$ where hybridized Dirac cone states arise [14]. Figure 2(a) shows the spectral function of $\mathrm{Bi} / \mathrm{Bi}_{2} \mathrm{Te}_{3}$ projected onto a $\mathrm{Bi}$ bilayer plus upper $1 \mathrm{QL} \mathrm{Bi}_{2} \mathrm{Te}_{3}$.
There appears only one Dirac cone at the $\Gamma$ point below Fermi level. Similar to the case of $\mathrm{Bi} / \mathrm{Bi}_{2} \mathrm{Se}_{3}$, three groups of data points are chosen around this Dirac cone, as marked by I, II, and III in Fig. 2(a). The corresponding momenta are -0.03 , -0.02 , and $-0.01 \AA^{-1}$ for I, II, and III states, respectively. The atom-projected components of these states are shown in Fig. 2(b), and the atomic layer indexes are the same as those shown in Fig. 1(c). One major difference from $\mathrm{Bi} / \mathrm{Bi}_{2} \mathrm{Se}_{3}$ is that the only one Dirac state in $\mathrm{Bi} / \mathrm{Bi}_{2} \mathrm{Te}_{3}$ has sizable contributions from both a $\mathrm{Bi}$ bilayer and $\mathrm{Bi}_{2} \mathrm{Te}_{3}$ substrate, as clearly seen from Fig. 2(b). This indicates that it is a hybridized Dirac state between the intrinsic surface Dirac state of $\mathrm{Bi}_{2} \mathrm{Te}_{3}$ and the extrinsic Rashba Dirac state of the Bi bilayer. A previous study has further shown another interesting point that such hybridization might enhance many-body interaction within the Dirac states [14]. Figure 2(b) also shows that the hybridization in the upper Dirac cone is stronger than that in the lower Dirac cone. Moving away from the Dirac point (from III to I), the components of the $\mathrm{Bi}$ bilayer $\left(\mathrm{Bi}_{2} \mathrm{Te}_{3}\right.$ substrate) decreases (increases) in the upper Dirac cone, but they show little change in the lower Dirac cone. The substrate contribution to the Dirac cone is not localized in the first QL but between the first and second QL [i.e., the sixth to ninth atomic layer as seen in Fig. 1(c)]. This is because the surface state is pushed slightly away from the $\mathrm{Bi} / \mathrm{Bi}_{2} \mathrm{Te}_{3}$ interface compared to the bare $\mathrm{Bi}_{2} \mathrm{Te}_{3}$ surface state [8]. The spin textures of this hybridized Dirac cone state along the iso-energy contour at the energy marked by label II are shown in Fig. 2(d). The total and orbit-resolved spin textures of the hybridized Dirac cone are the same as those in the intrinsic and extrinsic Dirac cones of the $\mathrm{Bi} / \mathrm{Bi}_{2} \mathrm{Se}_{3}$ 

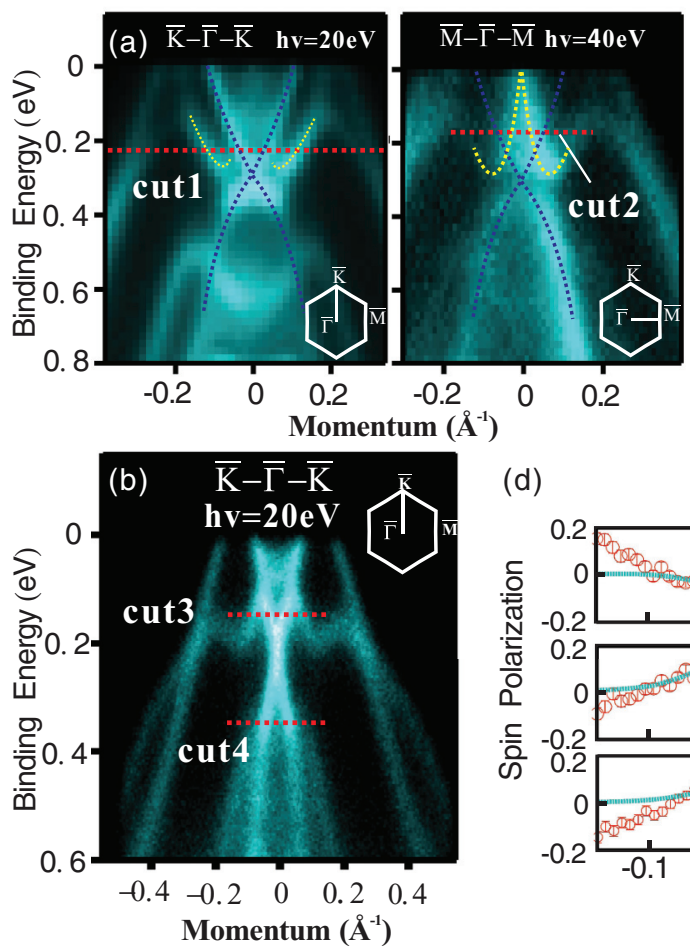

(d)
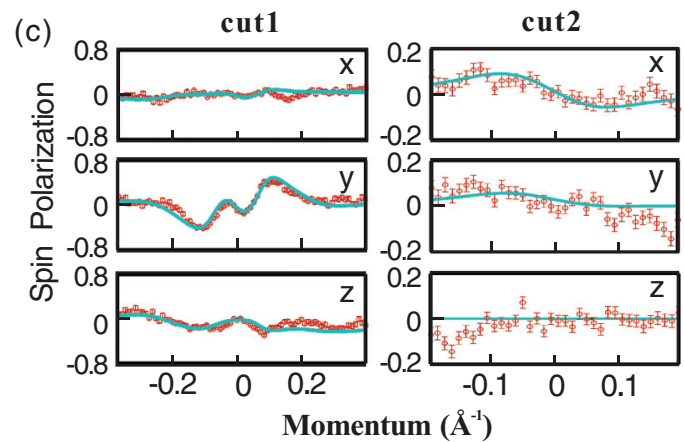

(e) cut1
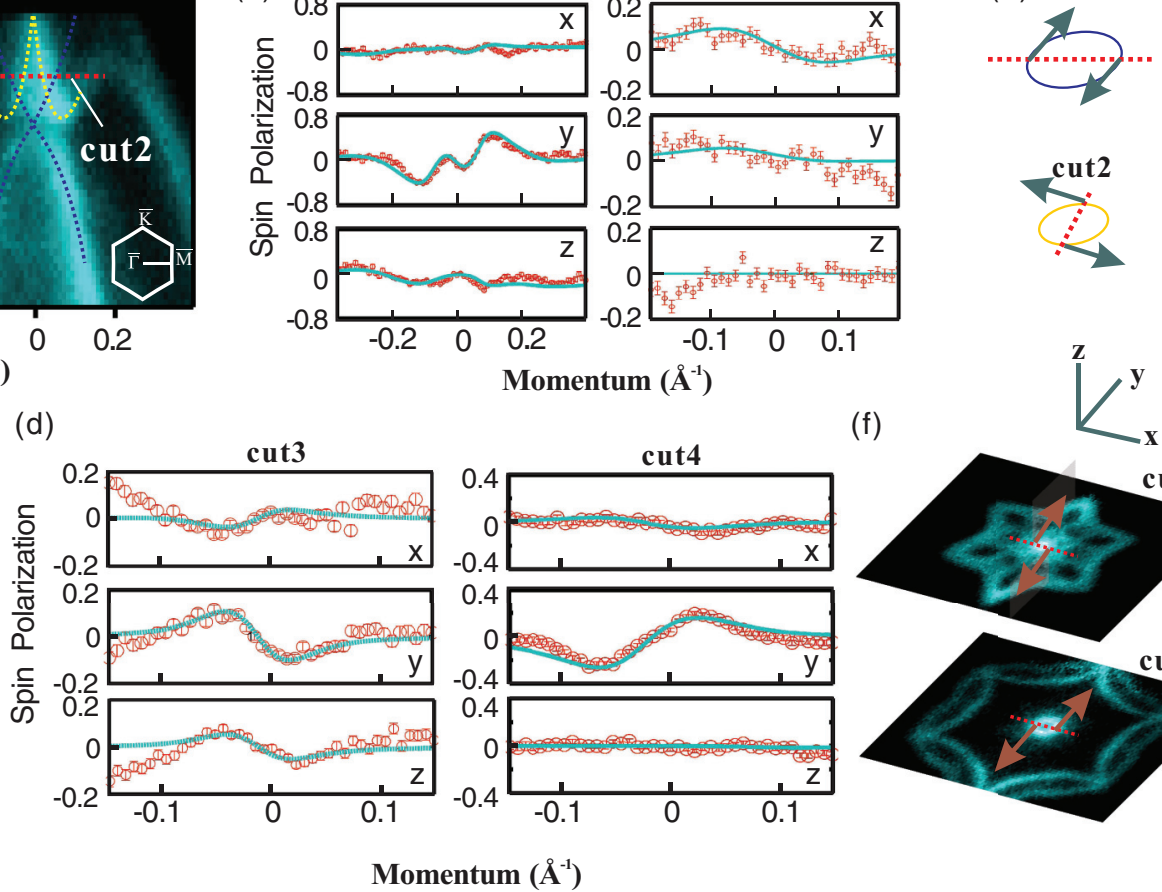

(f)
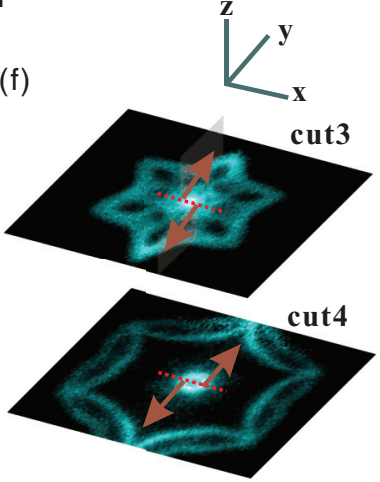

Momentum $\left(\AA^{-1}\right)$

FIG. 3. (Color online) (a) Spin-integrated ARPES spectra of $\mathrm{Bi} / \mathrm{Bi}_{2} \mathrm{Se}_{3}$ and (b) $\mathrm{Bi} / \mathrm{Bi}_{2} \mathrm{Te}_{3}$. The dashed blue and yellow lines show the position of the intrinsic and extrinsic Dirac cones. Experimentally, the relative intensity of two Dirac cones can be tuned by incident photon energy. (c) and (d) The experimental momentum dependent spin polarization and fitting data of $\mathrm{Bi}_{1} / \mathrm{Bi}_{2} \mathrm{Se}_{3}$ and $\mathrm{Bi} / \mathrm{Bi}_{2} \mathrm{Te}_{3}$. (e) and (f) $\mathrm{Spin}$ textures of intrinsic, extrinsic Dirac cones, and hybridized Dirac cone. The images in (f) are the constant energy contours of ARPES intensity.

system. $s$ and $p_{z}$ orbits have a helical spin texture, while $p_{x}$ and $p_{y}$ orbits have a nonhelical radial spin component.

We can conclude from the above calculation results several common features for the three different Dirac cone states. (1) The total spins have a helical spin texture, which is clockwise (counterclockwise) in the upper (lower) Dirac cone. (2) $s$ and $p_{z}$ orbit spins have the same texture as to the total spins. (3) $p_{x}$ and $p_{y}$ orbit spins have a radial spin component individually, but their sum becomes helical. (4) The substrate surface state is pushed down away from the first QL by the presence of the Bi bilayer.

To support our first-principles calculations, we have measured the total spin textures of these Dirac cone states in the $\mathrm{Bi} / \mathrm{Bi}_{2} \mathrm{Se}_{3}$ and $\mathrm{Bi} / \mathrm{Bi}_{2} \mathrm{Te}_{3}$ systems by SARPES. The method for epitaxially growing high quality $\mathrm{Bi} / \mathrm{Bi}_{2} \mathrm{Te}_{3}$ and $\mathrm{Bi} / \mathrm{Bi}_{2} \mathrm{Se}_{3}$ samples is the same as our previous works [14,21]. SARPES measurements were performed at the SIS beam line at the Swiss Light Source using the COPHEE spectrometer with two $40 \mathrm{kV}$ classical Mott detectors. The typical energy and momentum resolution was $50 \mathrm{meV}$ and $3 \%$ of the surface Brillouin zone at SIS. All the measurements were carried out at $30 \mathrm{~K}$ in ultrahigh vacuum with a base pressure better than $1 \times 10^{-10}$ torr. Proper measurement geometries were used to minimize SARPES matrix elements effects. For all measurements, $p$-polarized incident light was used to minimize the additional effect found in Ref. [10] as much as possible. For the $\mathrm{Bi} / \mathrm{Bi}_{2} \mathrm{Se}_{3}$ system, the relative spectra intensity of the two Dirac cone states can be tuned by using different photon energies. As shown in Fig. 3(a), the intrinsic Dirac cone of $\mathrm{Bi}_{2} \mathrm{Se}_{3}$ was observed clearly with photon energy of $20 \mathrm{eV}$ and the extrinsic Dirac cone of the Bi bilayer was detected with photon energy of $40 \mathrm{eV}$.

Figure 3(a) shows the spin-integrated ARPES spectra of $\mathrm{Bi} / \mathrm{Bi}_{2} \mathrm{Se}_{3}$ along $\bar{K}-\bar{\Gamma}-\bar{K}$ and $\bar{M}-\bar{\Gamma}-\bar{M}$ directions, in which one can see both intrinsic and extrinsic Dirac cones. In the SARPES measurements, two spin-resolved momentum distribution curves (MDC) were taken [cut1 and cut2, as shown by the horizontal dashed lines in Fig. 3(a)]. Cut1 is at $220 \mathrm{meV}$ below the Fermi level to study the intrinsic Dirac cone, and cut 2 is at $180 \mathrm{meV}$ below the Fermi level to study the extrinsic Dirac cone. The spin polarization is extracted by using the the two-step fitting routine of SARPES data [3,26,27]. The momentum dependent spin polarization and fitting data are shown in Fig. 3(c), from which we extract the spin textures. Figure 3(e) presents the spin textures of intrinsic and extrinsic Dirac cones, which is clockwise in the upper intrinsic Dirac cone and counterclockwise in the lower extrinsic Dirac cone. For both Dirac cones, spins are nearly in-plane polarized. Figure 3(b) shows the spin-integrated ARPES spectra of $\mathrm{Bi} / \mathrm{Bi}_{2} \mathrm{Te}_{3}$ along $\bar{K}-\bar{\Gamma}-\bar{K}$ direction, in which one can see the hybridized Dirac cone. Similar to the SARPES measurement for $\mathrm{Bi} / \mathrm{Bi}_{2} \mathrm{Se}_{3}$, we also take two MDC cuts (cut3 and cut4), as shown by the dashed lines in Fig. 3(b). Cut3 (cut4) is taken at 150 (350) meV below the Fermi level, slightly above (below) the hybridized Dirac cone. The polarization and fitting data are shown in Fig. 3(d). As shown in Fig. 3(f), the spin texture is clockwise and counterclockwise in the upper and lower Dirac cone, respectively. We note that using our experimental configuration, the complexity in SARPES measurements on TI materials [10] is largely suppressed. 
$\mathrm{Bi} / \mathrm{Bi}_{2} \mathrm{Se}_{3}$

(a)

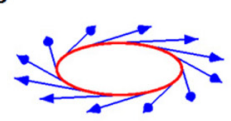

Bi-1
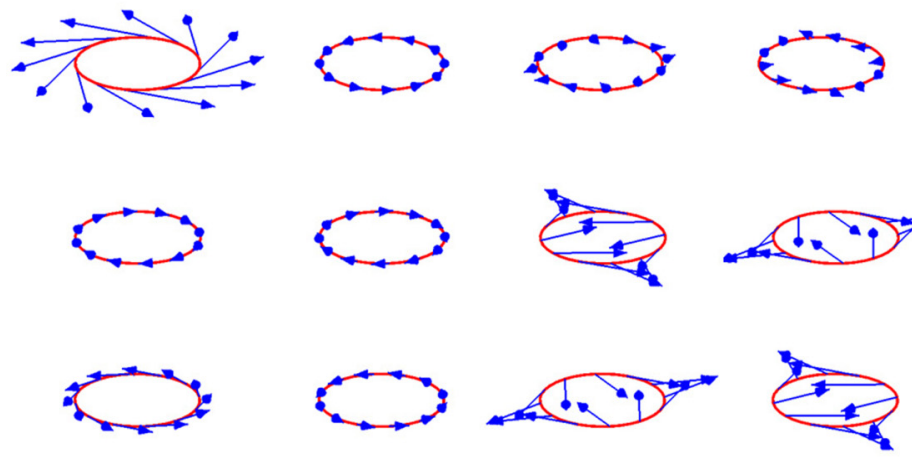

S
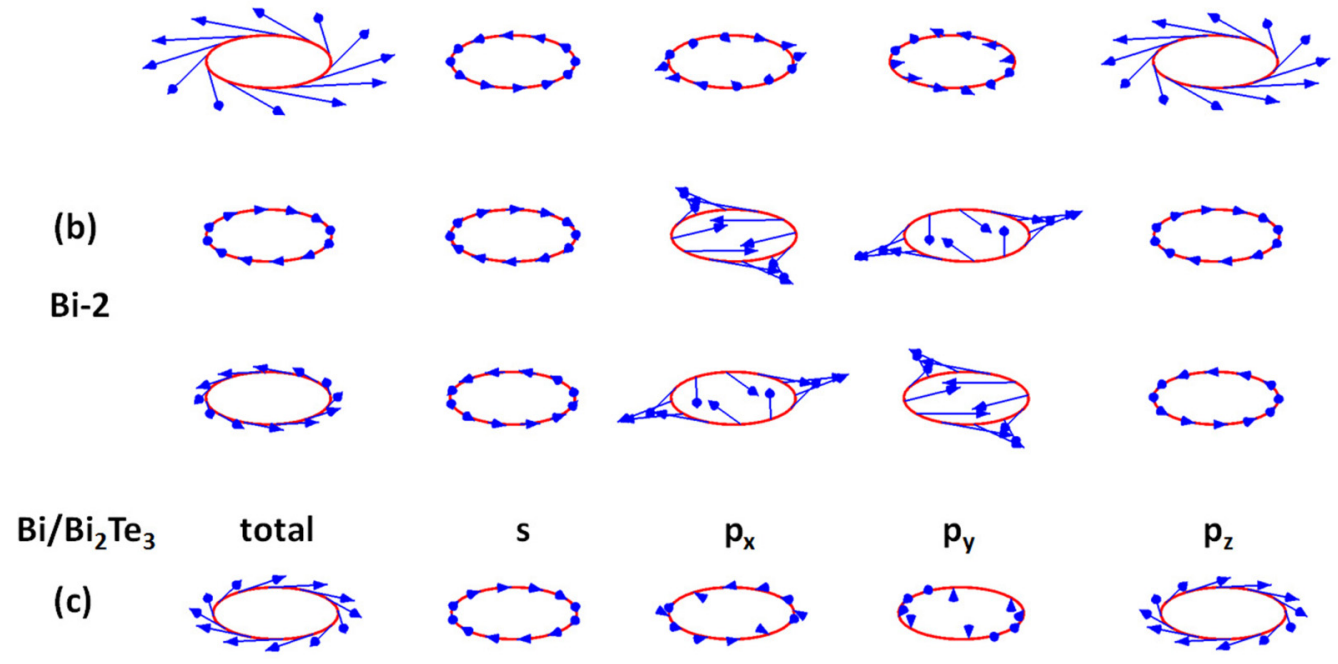

Bi-1
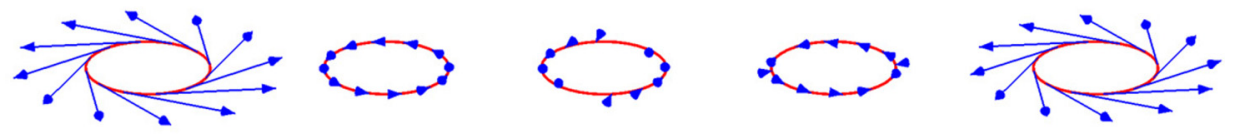

(d)
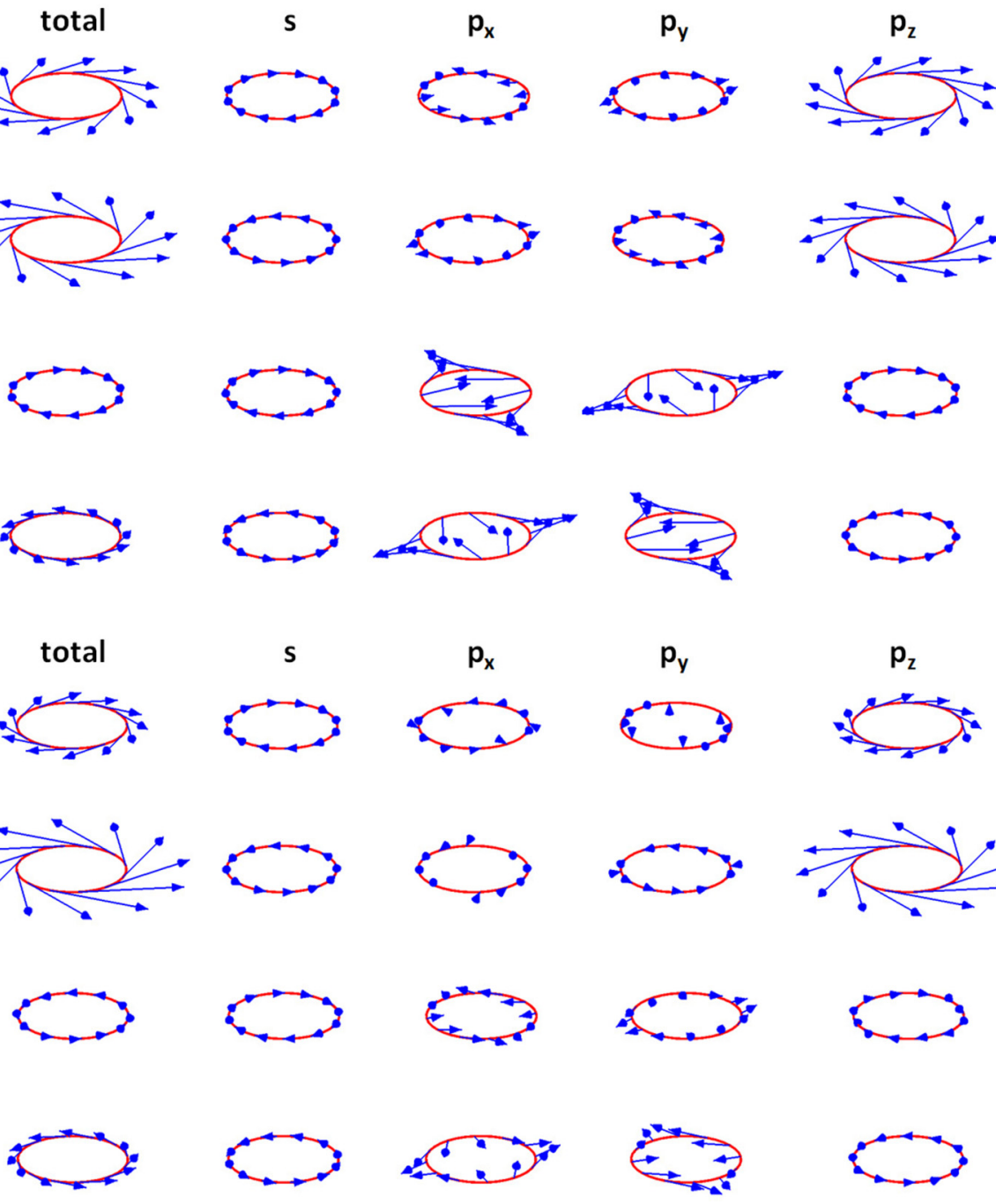

$p_{z}$
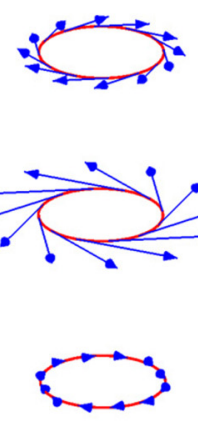

$\mathrm{Bi}-2$
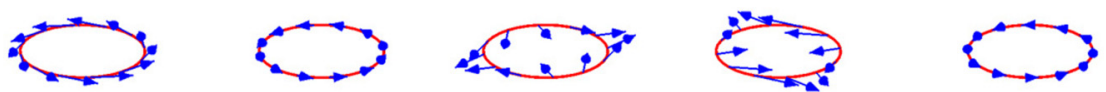

FIG. 4. (Color online) Atom- and orbit-resolved spin textures of the iso-energy contour Dirac cone state at the energy marked by label II in Figs. 1(a) and 2(a). (a) and (b) The spin textures of the first (Bi-1) and second (Bi-2) atom in $\mathrm{Bi} / \mathrm{Bi}_{2} \mathrm{Se}_{3}$. (c) and (d) The spin textures of the first (Bi-1) and second (Bi-2) atom in $\mathrm{Bi} / \mathrm{Bi}_{2} \mathrm{Te}_{3}$. The scaling factor of the arrow length is twice that in Fig. 1.

The measured spin orientation should mostly reflect the spin orientation in its initio states. Thus, to a large degree, our SARPES measurements indicated that all three Dirac cones have the same helical spin textures, independent of topology, consistent with our first-principle calculations.

Lastly, we present additional first-principles calculations to show the orbit- and atom-resolved spin textures of the Dirac cone states. Here we will focus on the top two Bi atoms. Overall, except the spin magnitude, the atom-resolved spin textures have the same structure compared to the spin textures by adding all atoms together, as shown in Fig. 4. However, from the atom projection, we can obtain some detailed local information of spin texture by considering the spin magnitude difference. Figures 4(a) and 4(b) are the spin textures of the extrinsic Dirac cone projected onto the top two $\mathrm{Bi}$ atoms of $\mathrm{Bi} / \mathrm{Bi}_{2} \mathrm{Se}_{3}$. We can see that the spin magnitude is very different between the two $\mathrm{Bi}$ atoms. For the $\mathrm{Bi}-1$ atom, $p_{z}$ orbit spin has the largest magnitude, while $s, p_{x}$, and $p_{y}$ orbit spins are negligible. But for the $\mathrm{Bi}-2$ atom, $p_{x}$ and $p_{y}$ orbit spins have the largest magnitude, while $s$ and $p_{z}$ orbit spins are negligible.
Figures 4(c) and 4(d) are the spin textures of the hybridized Dirac cone projected onto the top two $\mathrm{Bi}$ atoms of $\mathrm{Bi} / \mathrm{Bi}_{2} \mathrm{Te}_{3}$. The atom-resolved spin textures of the hybridized Dirac cone are similar to those in the extrinsic Dirac cone. We have also checked the atom-resolved spin textures for the intrinsic Dirac cone, which are again similar.

In summary, we have identified the helical spin textures for three different Dirac cone states in the interfaced systems of $\mathrm{Bi} / \mathrm{Bi}_{2} \mathrm{Se}_{3}$ and $\mathrm{Bi} / \mathrm{Bi}_{2} \mathrm{Te}_{3}$, from first-principles calculations and experiments. We confirm the recent theory [11] and experiment $[12,13]$ that the spin texture of intrinsic surface Dirac cone states of 3D TIs is coupled with orbit, resulting in an overall spin-orbit texture. Characteristically, $s$ and $p_{z}$ orbits have the conventional helical spin texture; $p_{x}$ and $p_{y}$ orbits show individually radial spin component, while the sum of the two shows a total in-plane helical spins. We further show that the same spin texture is also applicable to the extrinsic Rashba Dirac cone states of the $\mathrm{Bi}(111)$ bilayer on a substrate as well as to the hybridized Dirac cone states between a TI surface state and a thin-film Rashba state. Therefore, we suggest that 
the unique spin-orbit texture of helical Dirac states pertains to SOC, but not necessarily to TIs.

The experimental work conducted at Shanghai Jiao Tong University is supported by National Basic Research Program of China Grants No. 2012CB927401, No. 2011CB921902, No. 2013CB921902, and No. 2011CB922200; National Natural Science Foundation of China Grants No. 91021002, No. 10904090, No. 11174199, No. 11134008, No. 11227404, and No. 11274228; and Shanghai Committee of Science and Technology, China Grants No. 10JC1407100, No. 10PJ1405700, No. 11PJ405200, No. 12JC1405300, and No. 13QH1401500.
The theoretical work conducted at University of Utah is supported by the office of Basic Energy Sciences, US Department of Energy, Grant No. DE-FG02-04ER46148. We also thank the CHPC at University of Utah and NERSC for providing the computing resources. D.Q. acknowledges additional support from the Top-notch Young Talents Program and the Program for Professor of Special Appointment (Eastern Scholar) at Shanghai Institutions of Higher Learning. Z.F.W. acknowledges additional support from NSF-MRSEC (Grant No. DMR-1121252). The spin-resolved ARPES experiments were performed on the SIS beamline at the Swiss Light Source, Paul Scherrer Institut, Villigen, Switzerland.
[1] M. Z. Hasan and C. L. Kane, Rev. Mod. Phys. 82, 3045 (2010).

[2] X.-L. Qi and S.-C. Zhang, Rev. Mod. Phys. 83, 1057 (2011).

[3] D. Hsieh, Y. Xia, L. Wray, D. Qian, A. Pal, J. H. Dil, J. Osterwalder, F. Meier, G. Bihlmayer, C. L. Kane, Y. S. Hor, R. J. Cava, and M. Z. Hasan, Science 323, 919 (2009).

[4] D. Hsieh, Y. Xia, D. Qian, L. Wray, J. H. Dil, F. Meier, J. Osterwalder, L. Patthey, J. G. Checkelsky, N. P. Ong, A. V. Fedorov, H. Lin, A. Bansil, D. Grauer, Y. S. Hor, R. J. Cava, and M. Z. Hasan, Nature (London) 460, 1101 (2009).

[5] P. Roushan, J. Seo, C. V. Parker, Y. S. Hor, D. Hsieh, D. Qian, A. Richardella, M. Z. Hasan, R. J. Cava, and A. Yazdani, Nature (London) 460, 1106 (2009).

[6] Z.-H. Pan, E. Vescovo, A. V. Fedorov, D. Gardner, Y. S. Lee, S. Chu, G. D. Gu, and T. Valla, Phys. Rev. Lett. 106, 257004 (2011).

[7] S. Souma, K. Kosaka, T. Sato, M. Komatsu, A. Takayama, T. Takahashi, M. Kriener, K. Segawa, and Y. Ando, Phys. Rev. Lett. 106, 216803 (2011).

[8] S. V. Eremeev, G. Landolt, T. V. Menshchikova, B. Slomski, Y. M. Koroteev, Z. S. Aliev, M. B. Babanly, J. Henk, A. Ernst, L. Patthey, A. Eich, A. A. Khajetoorians, J. Hagemeister, O. Pietzsch, J. Wiebe, R. Wiesendanger, P. M. Echenique, S. S. Tsirkin, I. R. Amiraslanov, J. H. Dil, and E. V. Chulkov, Nat. Commun. 3, 635 (2012).

[9] C.-H. Park and S. G. Louie, Phys. Rev. Lett. 109, 097601 (2012).

[10] C. Jozwiak, C.-H. Park, K. Gotlieb, C. Hwang, D.-H. Lee, S. G. Louie, J. D. Denlinger, C. R. Rotundu, R. J. Birgeneau, Z. Hussain, and A. Lanzara, Nat. Phys. 9, 293 (2013).

[11] H. Zhang, C.-X. Liu, and S.-C. Zhang, Phys. Rev. Lett. 111, 066801 (2013).

[12] Y. Cao, J. A. Waugh, X.-W. Zhang, J.-W. Luo, Q. Wang, T. J. Reber, S. K. Mo, Z. Xu, A. Yang, J. Schneeloch, G. D. Gu, M. Brahlek, N. Bansal, S. Oh, A. Zunger, and D. S. Dessau, Nat. Phys. 9, 499 (2013).
[13] Z. Xie, S. He, C. Chen, Y. Feng, H. Yi, A. Liang, L. Zhao, D. Mou, J. He, Y. Peng, X. Liu, Y. Liu, G. Liu, X. Dong, L. Yu, J. Zhang, S. Zhang, Z. Wang, F. Zhang, F. Yang et al., Nat. Commun. 5, 3382 (2014).

[14] L. Miao, Z. F. Wang, W. Ming, M.-Y. Yao, M.-X. Wang, F. Yang, Y. R. Song, F. Zhu, A. V. Fedorov, Z. Sun, C. L. Gao, C. Liu, Q.-K. Xue, C.-X. Liu, F. Liu, D. Qian, and J.-F. Jia, Proc. Natl. Acad. Sci. USA 110, 2758 (2013).

[15] Z. F. Wang, M.-Y. Yao, W. Ming, L. Miao, F. Zhu, C. Liu, C. L. Gao, D. Qian, J.-F. Jia, and F. Liu, Nat. Commun. 4, 1384 (2013).

[16] T. Hirahara, G. Bihlmayer, Y. Sakamoto, M. Yamada, H. Miyazaki, S.-i. Kimura, S. Blügel, and S. Hasegawa, Phys. Rev. Lett. 107, 166801 (2011).

[17] S. Murakami, Phys. Rev. Lett. 97, 236805 (2006).

[18] Z. Liu, C.-X. Liu, Y.-S. Wu, W.-H. Duan, F. Liu, and J. Wu, Phys. Rev. Lett. 107, 136805 (2011).

[19] L. Chen, Z. F. Wang, and F. Liu, Phys. Rev. B 87, 235420 (2013).

[20] Z. F. Wang, L. Chen, and F. Liu, arXiv:1402.6754.

[21] F. Yang, L. Miao, Z. F. Wang, M.-Y. Yao, F. Zhu, Y. R. Song, M.-X. Wang, J.-P. Xu, A. V. Fedorov, Z. Sun, G. B. Zhang, C. Liu, F. Liu, D. Qian, C. L. Gao, and J.-F. Jia, Phys. Rev. Lett. 109, 016801 (2012).

[22] G. Kresse and J. Hafner, Phys. Rev. B 47, 558 (1993).

[23] Y. Zhang, K. He, C.-Z. Chang, C.-L. Song, L.-L. Wang, X. Chen, J.-F. Jia, Z. Fang, X. Dai, W.-Y. Shan, S.-Q. Shen, Q. Niu, X.-L. Qi, S.-C. Zhang, X.-C. Ma, and Q.-K. Xue, Nat. Phys. 6, 584 (2010).

[24] W. Zhang, R. Yu, H.-J. Zhang, X. Dai, and Z. Fang, New J. Phys. 12, 065013 (2010).

[25] G. Wu, H. Chen, Y. Sun, X. Li, P. Cui, C. Franchini, J. Wang, X.-Q. Chen, and Z. Zhang, Sci. Rep. 3, 1233 (2013).

[26] F. Meier, H. Dil, J. Lobo-Checa, L. Patthey, and J. Osterwalder, Phys. Rev. B 77, 165431 (2008).

[27] J. H. Dil, J. Phys.: Condens. Matter 21, 403001 (2009). 